\title{
Low Frequency Noise Measurement and Analysis of Capacitive Micro-Accelerometers: Temperature Effect
}

\author{
F. Mohd-Yasin ${ }^{\mathrm{a}}$, D.J. Nagel ${ }^{\mathrm{b}}$, D.S. Ong ${ }^{\mathrm{a}}$, C.E. Korman ${ }^{\mathrm{b}}$, H.T. Chuah ${ }^{\mathrm{a}}$ \\ ${ }^{a}$ Faculty of Engineering, Multimedia University, Cyberjaya, 63100 Malaysia \\ Phone:+603-83125423. E-mail: faisal.yasin@mmu.edu.my \\ ${ }^{\mathrm{b}}$ ECE Dept, The George Washington University, Washington DC, 20052 USA
}

Key words: accelerometers, noise, acceleration-dependent, temperature-dependence

Micro-accelerometer is one of the MEMS devices that has been used widely. Such accelerometers typically contain some movable capacitive micro-beams that respond to accelerations in one or more orthogonal directions. The ultimate performance of these devices is limited by the intrinsic noise within the device, mainly due to the combined effect of thermally dependent electrical and mechanical noise sources. Noise from any source limits the utility of MEMS accelerometers in low $\mathrm{g}$ and high accuracy situations, for example, for inertial navigation. Understanding and controlling the relative importance and interactions of electrical and mechanical noise are intellectually challenging problems.

Over the years, a number research groups had modeled and characterized noise of microaccelerometers but none of them investigated the dependence of noise on acceleration. Levinzon [1] presented the fundamental noise limit of piezoelectric accelerometers based on the mechanical-thermal and electrical-thermal noise. Yoshida et al. [2] proposed a linear noise model which does not include the thermal-mechanical characteristics in the circuit noise. They found that the simulated noise spectrum is generally matched to the measured noise from capacitive-servo accelerometer. Rocha at al. [3] attempted to characterize directly the mechanical-thermal noise spectrum by repeatedly bringing the capacitive micro sensor to pull-in, and measuring the pull-in time followed by FFT. In all three groups, the accelerometer was custom-made.

This group performed the Low Frequency Noise Measurement (LFNM) technique (Figure 1) to study noise characteristics of commercial accelerometers with single and dual axis [4]-[5]. The noise spectrum was measured as a function of the acceleration of gravity in the range from -1 to $+1 \mathrm{~g}$. A common spectral behavior of noise was found, with $1 / f$ noise dominating at low frequencies and white thermal noise being the limiting factor at higher frequencies. An acceleration dependence of the noise was found in $\mathrm{AD}$ and Freescales accelerometers, in contrast to prevailing theory [6]. The theory governing the behavior of noise in MEMS accelerometers predicts that both electrical and mechanical noise should vary as the square root of the temperature. In this work, we present a noise measurement incorporating temperature effect. In the setup, a hot plate is used to heat the device up to temperatures in the 75 degree $\mathrm{C}$ range. A thermo couple attached to the enclosure is used to assess its temperature. The noise characteristics of $\mathrm{AD}$ and Freescales micro-accelerometers with temperature effect are shown in Figure 2 and 3, respectively. The data show that the noise power increases at high temperature, but not as significant as predicted by the theory.

References:

[1] F.A. Levinzon "Fundamental Noise Limit of Piezoelectric Accelerometer", IEEE Sensors Journal, Vol. 4, No. 1, pp 108-111, Feb 2004.

[2] Y. Yoshida, H. Kakuma, H. Asanuma, H. Niitsuma "A Linear Model Based Noise Evaluation of a Capacitive Servo Accelerometer Fabricated by MEMS”, IEICE ELEX, Vol. 2, No. 6, 198-205, 2005.

[3] L.A. Rocha, E. Cretu, R.F. Wolffenbuttel "Measuring and Interpreting the Mechanical Thermal Noise in MEMS", J. Micromech. Microeng, Vol. 15, S30-38, 2005.

[4] F. Mohd-Yasin, C.E. Korman, D.J. Nagel "Measurement of Noise Characteristics of MEMS Accelerometers", Solid State Electronics Journal, Vol/Issue: 42/2, pp. 357-360, 2003.

[5] F. Mohd-Yasin, D.J. Nagel, D.S. Ong, C.E. Korman, H.T. Chuah "Low Frequency Noise Measurement

And Analysis Of Capacitive Micro-Accelerometers", Microelectronics Engineering, in press.

[6] T.B. Gabrielson, "Mechanical-Thermal Noise in Micromachined Acoustic and Vibration sensors", IEEE Transactions on Electron Devices, Vol. 40, No. 5. pp. 903-909, May 1993. 
Low Frequency Noise Measurement and Analysis of Capacitive Micro-Accelerometers:

Temperature Effect

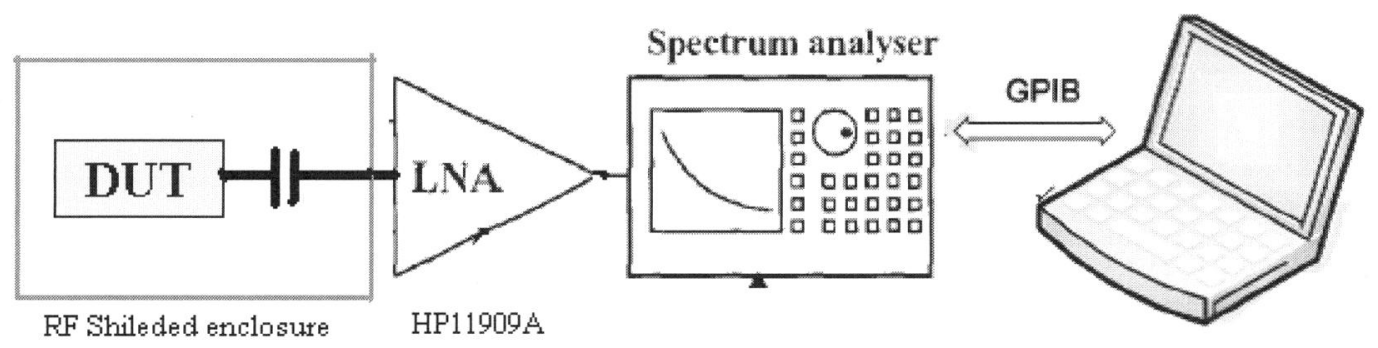

Figure 1: Low Frequency Noise Measurement Setup

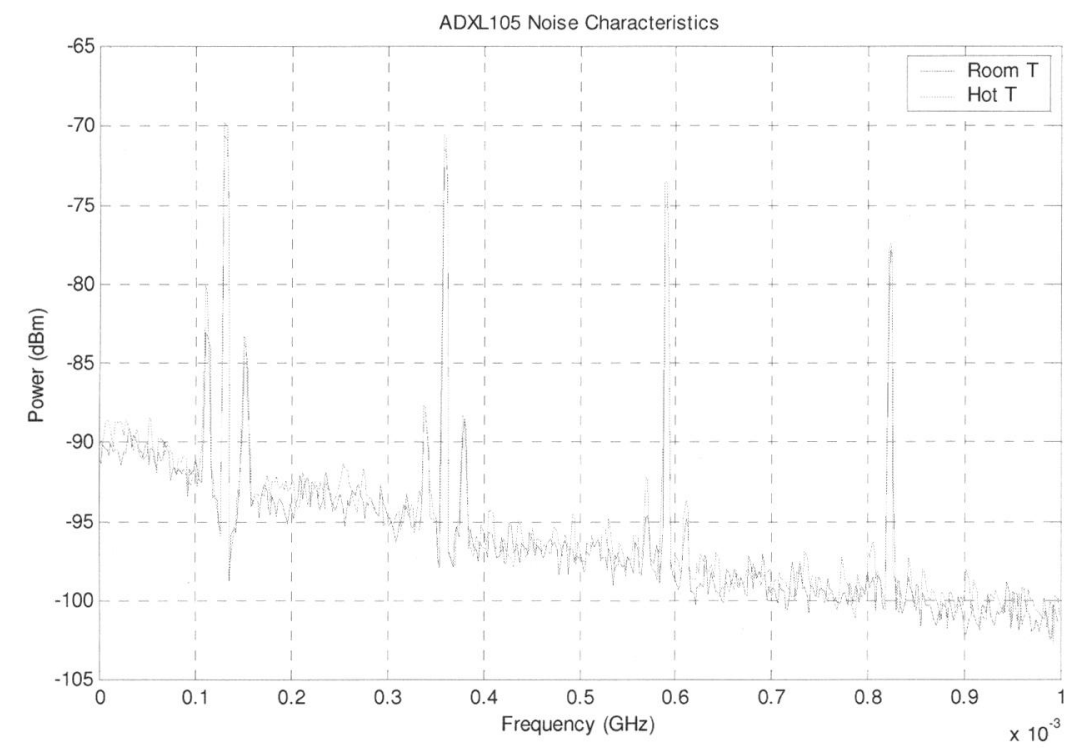

Figure 2: ADXL105 noise characteristics at room and high temperatures

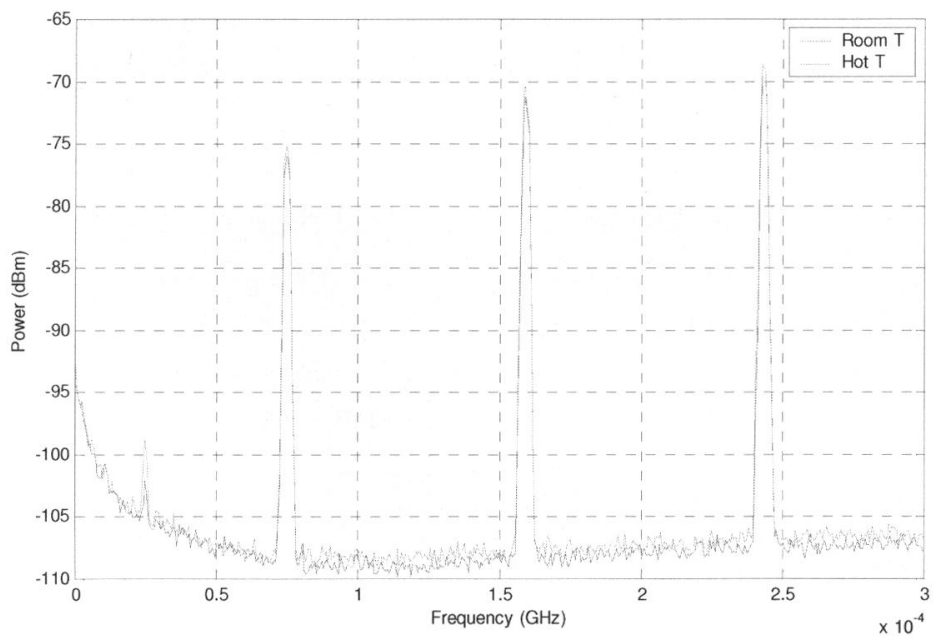

Figure 3: Freescales 3201 noise characteristics at room and high temperatures 